\title{
Lithography method for selective area of CNTs growth
}

\begin{abstract}
This paper presents the processes of fabrication method in the selective area of growth Carbon Nanotubes (CNTs) on the substrate with an Interdigitated (IDE) electrodes pattern using resist AZ1500. The substrate used in this work was Gallium Phosphate with Chromium $(0.021 \mathrm{\varepsilon m})$ and Platinum $(0.11 \mathrm{\varepsilon m})$ as the metal layer. The CNTS was grown in two different temperatures using chemical vapor deposition (CVD) with hydrogen as the process gas and benzene as the hydrocarbon. The most suitable temperature growth for CNTs in this work was found to be $800^{\circ} \mathrm{C}$. In this study, CNTs were produced by CVD impregnated with iron nitrate $(\mathrm{Fe}(\mathrm{NO} 3) 3.9 \mathrm{H} 2 \mathrm{O})$ solution and Resist AZ1500 as the mask for the selective area grown. Maximum temperature for Resist AZ1500 was at $120^{\circ} \mathrm{C}$. Therefore Iron Nitrate was used as the protector to protect the resist to be evaporated. The Resist AZ1500 and the Iron Nitrate were coated in different layer on the substrate using standard lithography method.
\end{abstract}

